\title{
Is fibroid location associated with hemorrhage and complication rates following laparoscopic myomectomy?
}

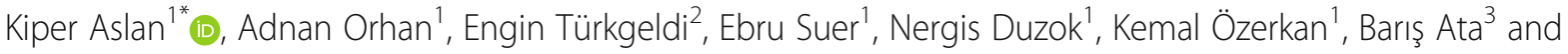
Gürkan Uncu'

\begin{abstract}
Objective: To determine whether hemorrhage and complication rates vary according to the location of the dominant fibroid following laparoscopic myomectomy.

Background: Laparoscopic myomectomy is associated with less postoperative pain, analgesic requirement, shorter hospitalization period, and less febrile complications when compared to conventional laparotomy. Despite the advantages, complications like hemorrhage, blood transfusion, bowel and urinary tract injury, and conversion to laparotomy may be seen in laparoscopic myomectomy. We do not know whether the fibroid location affects these complications.
\end{abstract}

Materials and methods: Women, who underwent laparoscopic myomectomy at two different tertiary academic hospitals, were analyzed retrospectively. Only women with at least one intramural fibroid (Monroe type 3, 4, or 5) were included. Patients were categorized according to localization of the dominant fibroid, i.e., anterior uterine wall, posterior uterine wall, and fundus. Change in hemoglobin levels before and after surgery and complication rates were compared across categories.

Results: Two hundred nineteen women with a mean age of $35.7 \pm 6$ years were included. There were 81 women with fundal fibroid, 56 with anterior wall, and 72 with posterior wall fibroid. The other ten women with intraligamentary and isthmic fibroid were excluded. The mean fibroid diameter was $6.7 \pm 2.6,6.6 \pm 2.3$, and $6.7 \pm$ $2.3 \mathrm{~cm}$ in the fundal, anterior, and posterior groups, respectively $(p=0.9)$. The median (25th-75th percentile) changes in hemoglobin levels were $1.5(0.8-2.2), 1.3(0.6-2.1)$, and $1.3(0.9-2) \mathrm{g} / \mathrm{dl}$ in fundal, anterior, and posterior wall groups, respectively $(p=0.55)$. There were $5(6.2 \%), 5(8.9 \%)$, and $2(2.8 \%)$ complications in fundal, anterior, and posterior wall groups, respectively $(p=0.33)$.

Conclusion: The incidence of hemorrhage or complication does not seem to vary depending on the fibroid location. However, the sample size was limited; observed values suggest that fibroid location does not affect hemorrhage and complication rates.

Keywords: Myomectomy, Laparoscopy, Leiomyoma, Fibroid

\footnotetext{
* Correspondence: kiperaslan@yahoo.com.tr

'Department of Obstetrics and Gynecology, Uludag University School of

Medicine, Gorukle, Bursa, Turkey

Full list of author information is available at the end of the article
}

\section{Springer Open}

(c) The Author(s). 2020 Open Access This article is licensed under a Creative Commons Attribution 4.0 International License, which permits use, sharing, adaptation, distribution and reproduction in any medium or format, as long as you give appropriate credit to the original author(s) and the source, provide a link to the Creative Commons licence, and indicate if changes were made. The images or other third party material in this article are included in the article's Creative Commons licence, unless indicated otherwise in a credit line to the material. If material is not included in the article's Creative Commons licence and your intended use is not permitted by statutory regulation or exceeds the permitted use, you will need to obtain permission directly from the copyright holder. To view a copy of this licence, visit http://creativecommons.org/licenses/by/4.0/. 


\section{Background}

Fibroids are the most common benign tumors of the female genital system, and $25-30 \%$ of women are diagnosed with a fibroid during their lifetime [1]. While fibroids can be asymptomatic, some patients present with abdominal discomfort or pain, abnormal uterine bleeding, anemia, or subfertility depending on the size and location of the fibroid among other factors [2]. While asymptomatic fibroids can be managed expectantly, symptomatic fibroids may require surgical treatment. Medical treatment may also be effective in reducing the number of women who required surgery. Currently ulipristal acetate (selective progesterone receptor modulator) has been suggested to reduce the fibroid size and the symptoms. In a multicenter study, it is shown that using ulipristal acetate may reduce the symptoms in $57 \%$ in symptomatic women and provide the treatment without surgery [3]. Although medical approach, most symptomatic women may undergo surgery. Compared to conventional myomectomy with laparotomy, laparoscopic myomectomy is associated with less postoperative pain, analgesic requirement, shorter hospitalization period, and less febrile complications [4]. Despite the advantages, complications like hemorrhage, blood transfusion, bowel and urinary tract injury, and conversion to laparotomy may be seen in laparoscopic myomectomy.

Even though most fibroids can be excised with laparoscopy, opinions vary regarding patient selection. Fibroid location and size are factors that are invariably considered to determine eligibility. Although the presence of an association between fibroid location and surgical complications is logical and straightforward, a quantitative assessment of this association would be helpful for better patient selection for laparoscopic myomectomy.

\section{Materials and methods}

This was a multicenter retrospective study conducted at the Department of Obstetrics and Gynecology of Uludag University Faculty of Medicine Hospital, Bursa, and Department of Obstetrics and Gynecology of Koc University School of Medicine, Istanbul, Turkey. Uludag University Institutional Review Board approved the study protocol with the ethics committee number 2014$12 / 10$. We screened our electronic records to identify patients who underwent laparoscopic myomectomy between January 2013 and January 2018. All patients underwent a systematic preoperative assessment including medical history, pelvic examination, gynecologic ultrasonography, and laboratory assessment. The location and size of fibroids were documented by imaging methods including transvaginal and transabdominal ultrasonography or an abdominal MRI prior to the operation. Only women with at least one intramural fibroid (Munroe type 3, 4, or 5) were included in the analysis.

All operations were performed under general anesthesia. Patients were placed in dorsal lithotomy position, a Foley catheter was inserted to the urinary bladder, and a uterine manipulator was fixed on the cervix. Pneumoperitoneum was achieved with insufflation of 3-3.5 lt of $\mathrm{CO}_{2}$ insufflated with a Veress needle inserted through the umbilicus. A $10-\mathrm{mm}$ port was placed intra-umbilically for telescope, a $10-\mathrm{mm}$ port was placed approximately $3-4 \mathrm{~cm}$ medial to the left superior iliac spine, and a 5-mm port was placed through the left superior quadrant at the level of or slightly below umbilicus. The location of ancillary ports depended on the location and size of fibroids. Following exploration of the abdomen and examination of the uterus, an incision over the dome of the fibroid was done using a harmonic scalpel. For anterior wall fibroids, the incision line was parallel to the longitudinal axis of the uterus, whereas a transverse or oblique incision was preferred for fundal or posterior wall fibroids. Sharp dissection was continued until identification of the plane between the fibroid and its pseudocapsule. Fibroid(s) was enucleated from its bed by traction and counter traction. After enucleation, the fibroid bed was inspected for bleeders and bipolar coagulation was applied as deemed necessary by the surgeon. There was not any intra-myometrial medication or aqua dissection used for less hemorrhage. The fibroid bed was sutured in a transverse, continuous, and non-locking fashion with no. 1 Vicryl or no.0 V-Lock suture in 1 or 2 layers depending on the depth of fibroid bed. Copious irrigation and lavage was done to check hemostasis. Fibroids were morcellated (Karl Storz, Germany) through a $10-\mathrm{mm}$ sleeve. All operations were performed by the same advanced surgeons. All the patients were up and about within $6 \mathrm{~h}$ of surgery, were orally allowed, and were discharged the next morning.

Patients' age, fibroid location (categorized as fundal, posterior wall, anterior wall), size of the largest fibroid, number of the fibroids, change in hemoglobin concentration from before surgery to postoperative day 1 , and complications (conversion to laparotomy, excessive bleeding requiring blood transfusion, residual fibroid) were collected from electronic medical records.

Distribution of data was assessed with histograms. Depending on distribution characteristics, continuous variables were defined with mean (standard deviation) or median (25th and 75 th percentiles). Categorical variables were defined as percentages. One-way analysis of variance (ANOVA), Kruskal-Wallis, chi-square test, and its derivatives were used for between-group comparisons. A two-tailed $p$ value $<0.05$ was considered as statistically significant. 


\section{Results}

A total of 219 women with mean age of $35.7 \pm 6$ years were included. Mean fibroid diameter was $6.7 \pm 2.3 \mathrm{~cm}$. There were 81 women with fundal, 56 with anterior wall, and 72 with posterior wall fibroids. All these fibroids were intramurally located. One woman with an isthmic, 8 women with intraligamentary, and one woman with cervical fibroid were excluded from comparative analysis. Age, preoperative hemoglobin levels, fibroid size, and numbers in each group are presented in Table 1. There were no significant differences between the groups for these variables. Change in hemoglobin concentration and complication rates are presented in Table 2. In 46 patients, there was no need to determine postoperative hemoglobin values because of minimal hemorrhage, so change in hemoglobin concentrations was analyzed in 173 patients and values were likewise similar between the groups. One patient in the fundal fibroid group was diagnosed with a residual fibroid after surgery, two patients in the fundal fibroid group and five patients in the corpus anterior group required blood transfusion. Three patients in fundal fibroid and one patient in the corpus posterior fibroid group required conversion to laparotomy due to bleeding during surgery. One patient in the corpus posterior fibroid group had severe vaginal bleeding after discharge and required rehospitalization. The low absolute numbers in complications prevented a reliable statistical comparison, but there was no obvious trend towards an increased complication rate in any of the groups.

\section{Discussion}

Abdominal myomectomy was first introduced by Boney in 1931 [5], and it had been the only surgical procedure for years until endoscopic techniques were described. Abdominal myomectomy is still a proper procedure for multiple and giant myomas. Semm described laparoscopic myomectomy in 1979 for subserosal myomas [6]. In the beginning of the 1990s, laparoscopic myomectomy began to be performed in intramural myomas. Laparoscopic myomectomy has less hemorrhage, fewer adhesions, and shorter recovery time when compared with laparotomic myomectomy. In a retrospective study with 1001 patients, Paul and colleagues showed that hemorrhage and blood transfusion are the most seen complications in laparoscopic myomectomy [7]. In a prospective study including 2050 laparoscopic myomectomy between the years 1998 and 2004, the overall complication rate was found $11.1 \%$. The most seen complication was hemorrhage, and mean hemoglobin drop was $1.5 \mathrm{~g} / \mathrm{dl}$ [8]. In a study about predictors of hemorrhage in laparoscopic myomectomy; the hemorrhage risk was found associated with the duration of surgery, the largest diameter of fibroid, the cumulative fibroid weight, and the number of the suture [9]. There are numerous studies about the prediction of hemorrhage risk in laparoscopic myomectomy, but none of them mention whether the location of fibroid is associated with the risk of hemorrhage or other complications. Based on these concerns, in our study, we planned to detect whether hemorrhage and complication rates vary according to the location of the dominant fibroid. In our study, the median fibroid size was $6.7 \mathrm{~cm}$, similar with Sizzi's large case serial $(6.4 \mathrm{~cm})$ [8]. Our median hemoglobin drop was $1.5 \mathrm{~g} / \mathrm{dl}$ in the fundal group, $1.3 \mathrm{~g} /$ $\mathrm{dl}$ in the anterior group, and $1.3 \mathrm{~g} / \mathrm{dl}$ in the posterior fibroid group. The results were similar with other studies. Except for four patients (laparo-conversion), all the myomas were enucleated by laparoscopy, and all the patients had no severe bleeding. There was no bowel or urinary tract injury.

There are two factors associated with hemoglobin drop in LM. These are appropriate dissection cleavage during myomectomy and fast suturation after myomectomy. If the surgeon could not find the fibroid capsule properly, there would be more hemorrhage during dissection. Finding the proper cleavage may be difficult in some degenerated fibroids. The second process of the operation is suturing the myomectomy incision. Suturation may be difficult depending on the location and size of fibroid. Also, if the surgeon is not advanced in laparoscopy, suturation time may be longer and it may cause more hemorrhage during the operation. If the surgeon is not advanced, there may be a need for ancillary port to make the operation with less hemorrhage. In our study in laparoscopy, we could not find the number of operations made with ancillary port.

The primary aim of the study was to obtain whether the fibroid location affects the hemorrhage rates. As the result, bleeding does not seem to vary depending on fibroid location. Although we were not able to conduct a reliable statistical comparison of complication rates due

Table 1 Demographic data of patients

\begin{tabular}{|c|c|c|c|c|}
\hline & Fundal $(n=81)$ & Anterior $(n=56)$ & Posterior $(n=72)$ & $p$ \\
\hline Age & $36.7+5.4$ & $34.3+6.5$ & $35.5+6.1$ & 0.172 \\
\hline Median number of fibroids (25th-75th percentile) & $2(1-2)$ & $1(1-3)$ & $1(1-2)$ & 0.208 \\
\hline Size in cm (SD) & $6.7(2.6)$ & $6.6(2.3)$ & $6.7(2.3)$ & 0.983 \\
\hline Mean preoperative $\mathrm{Hb}$ in $\mathrm{g} / \mathrm{dl}$ (SD) & $12.0(1.69)$ & $11.5(1.7)$ & $12.2(1.7)$ & 0.09 \\
\hline
\end{tabular}


Table 2 Hemorrhage and complication rates

\begin{tabular}{|c|c|c|c|c|}
\hline & Fundal $(n=81)$ & Anterior $(n=56)$ & Posterior $(n=72)$ & $p$ \\
\hline Median $\mathrm{Hb}$ change in $\mathrm{g} / \mathrm{dl}$ (25th-75th percentile) & $1.5(0.8-2.2)$ & $1.3(0.6-2.1)$ & $1.3(0.9-2)$ & 0.55 \\
\hline Complication & $6.2 \%(5 / 81)^{*}$ & $8.9 \%(5 / 56)$ & $2.8 \%(2 / 72)^{* *}$ & 0.33 \\
\hline Transfusion & $2.5 \%(2 / 81)$ & $8.9 \%(5 / 56)$ & 0 & \\
\hline Laparoconversion & $3.7 \%(3 / 81)$ & 0 & $1(\% 1.4)$ & \\
\hline
\end{tabular}

${ }^{*}$ One patient has residual fibroid after surgery

${ }^{* *}$ One patient has severe vaginal bleeding after discharge

to small sample size, the observed values suggest that intramural fibroids located in the anterior wall can be more prone to have more hemorrhage than fundal or posterior wall fibroids.

There are some limitations to our study. First, all the operations were performed by 6 surgeons. Although all of the surgeons are seniors on their departments, it is better to perform by one advanced surgeon for more reliable results. Another conflicting factor is the type of the suture material. It is widely known that using barbed suture during myomectomy makes the duration of operation shorter with less hemorrhage, and it is shown that using barbed suture has no negative impact on myomectomy scar healing in a multicenter study [10]. In our study, we used both types of the suture and we could not analyze the effect of the suture type on LM complications.

The other concerning issue is morcellation. The major risk in morcellation is malignancy potential of fibroids. As known, occult sarcoma or leiomyosarcoma incidence during myomectomy or hysterectomy is $0.39 \%$ [11]. Spillage or contamination of tissues during morcellation carriages risk of dissemination of tumoral cells through the abdomen and other organs, shortens the survey of survival, and increases the recurrence risk [12]. There are numerous case reports about disseminated leiomyosarcoma and sarcoma after uncontained power morcellation during myomectomy [13], and it is shown that uncontained power morcellation is associated with high risk of mortality in women with occult sarcoma [12]. There are novel containing methods during myomectomy or hysterectomy, like morcellation in plastic bags [14]. Although there are these novel containing methods, there is still suspicion of malignancy spillage during morcellation. In our study, we morcellated all the fibroids, and plastic bag was used depending on surgeon's choice. Plastic bag was used in approximately $30 \%$ of the cases. There was just 1 patient in 219 with unexpected pathology result with stump (smooth tumors with uncertain malignant potential). There was no recurrence during 6 years follow-up of this patient. Patient selection, age, and ultrasound findings are important factors for excluding malignancy, but occult sarcoma or leiomyosarcoma may be present even without any finding
[15]. Thus, laparoscopic myomectomy should be performed under contained morcellation according to the novel studies. But recently, surgeons leave laparoscopic myomectomy because of these unclear issues, and laparotomy rates begin to increase. After FDA report against the use of power morcellation, Multinu et al. showed that laparotomy rates in hysterectomy/myomectomy operations significantly increased and minor complication rates increased 20\%. They advised balancing against the potential harms of morcellation during a shared decision-making process between clinician and patient [16].

\section{Conclusion}

In conclusion, our study is the first study which showed that fibroid location does not affect hemorrhage and complication rates. Other factors like fibroid size, suturation technique and material, and surgeon's experience may affect the hemorrhage and complication rates. Due to our small sample size and retrospective design, our results should be assessed by larger prospective trials.

\section{Acknowledgements}

Not applicable

\section{Authors' contributions}

Kiper Aslan M.D.: writing, analysis, interpretation of data. Adnan Orhan M.D.: surgeon. Engin Türkgeldi M.D.: surgeon. Ebru Suer M.D.: collecting data. Nergis Duzok M.D.: collecting data. Kemal Özerkan Prof. Dr.: surgeon. Barış Ata Prof. Dr.: surgeon, design of the work, revision. Gürkan Uncu Prof. Dr.: surgeon, revision. The authors read and approved the final manuscript.

Funding

There is no funding in this study.

Availability of data and materials

The datasets used and/or analyzed during the current study are available from the corresponding author on reasonable request.

Ethics approval and consent to participate

Bursa Uludag University Institutional Review Board approved the study protocol with the ethics committee number 2014-12/10.

\section{Competing interests}

The authors declare that they have no competing interests.

\section{Author details}

'Department of Obstetrics and Gynecology, Uludag University School of Medicine, Gorukle, Bursa, Turkey. 2Department of Obstetrics and Gynecology, 
Koc University Hospital, Istanbul, Turkey. ${ }^{3}$ Department of Obstetrics and Gynecology, Koc University School of Medicine, Istanbul, Turkey.

Received: 2 April 2020 Accepted: 12 May 2020

Published online: 26 May 2020

\section{References}

1. Mettler L, Schollmeyer T, Tinelli A et al (2012) Complications of uterine fibroids and their management, surgical management of fibroids, laparoscopy and hysteroscopy versus hysterectomy, haemorrhage, adhesions, and complications. Obstet Gynecol Int 2012:791248. https://doi. org/10.1155/2012/791248

2. Khaund A, Lumsden MA (2008) Impact of fibroids on reproductive function. Best Pract Res Clin Obstet Gynaecol 22:749-760. https://doi.org/10.1016/j. bpobgyn.2008.01.009

3. Giarrè G, Franchini M, Castellacci E et al (2020) Ulipristal acetate in symptomatic uterine fibroids. A real-world experience in a multicentric Italian study. Gynecol Endocrinol 36:171-174. https://doi.org/10.1080/ 09513590.2019 .1648419

4. Jin C, Hu Y, Chan CX et al (2009) Laparoscopic versus open myomectomy-a meta-analysis of randomized controlled trials. Eur J Obstet Gynecol Reprod Biol. 145:14-21

5. Kalogiannidis I, Prapas N, Xiromeritis P, Prapas Y (2010) Laparoscopically assisted myomectomy versus abdominal myomectomy in short-term outcomes: a prospective study. Arch Gynecol Obstet 281:865-870. https:// doi.org/10.1007/s00404-009-1187-9

6. Semm K (1979) New methods of pelviscopy (gynecologic laparoscopy) for myomectomy, ovariectomy, tubectomy and adnectomy. Endoscopy 11:85-93

7. Paul GP, Naik SA, Madhu KN, Thomas T (2010) Complications of laparoscopic myomectomy: a single surgeon's series of 1001 cases. Aust N Z J Obstet Gynaecol 50:385-390. https://doi.org/10.1111/j.1479-828X.2010. 01191.x

8. Sizzi O, Rossetti A, Malzoni M et al (2007) Italian multicenter study on complications of laparoscopic myomectomy. J Minim Invasive Gynecol 14: 453-462. https://doi.org/10.1016/j.jmig.2007.01.013

9. Watrowski R, Jager C, Forster J (2017) Predictors of postoperative hemoglobin drop after laparoscopic myomectomy. Wideochirurgia I Inne Tech Maloinwazyjne 12:81-87. https://doi.org/10.5114/wiitm.2017.66515

10. Tinelli R, Litta P, Angioni S et al (2016) A multicenter study comparing surgical outcomes and ultrasonographic evaluation of scarring after laparoscopic myomectomy with conventional versus barbed sutures. Int J Gynecol Obstet 134:18-21. https://doi.org/10.1016/j.ijgo.2015.10.029

11. Multinu F, Casarin J, Tortorella L et al (2019) Incidence of sarcoma in patients undergoing hysterectomy for benign indications: a populationbased study. Am J Obstet Gynecol 220:179.e1-179.e10. https://doi.org/10. 1016/j.ajog.2018.11.1086

12. Xu X, Lin H, Wright JD et al (2019) Association between power morcellation and mortality in women with unexpected uterine cancer undergoing hysterectomy or myomectomy. J Clin Oncol 37:3412-3424. https://doi.org/ 10.1200/JCO.19.00562

13. Nappi L, Sorrentino F, Angioni S et al (2016) Leiomyomatosis Peritonealis Disseminata (LPD) ten years after laparoscopic myomectomy associated with ascites and lymph nodes enlargement: a case report. Int J Surg Case Rep 25:1-3. https://doi.org/10.1016/j.jisscr.2016.05.017

14. Angioni S, Pontis A, Multinu A, Melis G (2016) Safe endobag morcellation in a single-port laparoscopy subtotal hysterectomy. Minim Invasive Ther Allied Technol 25:113-116. https://doi.org/10.3109/13645706.2015.1109521

15. Tchartchian G, Bojahr B, Becker S et al (2019) Occult malignancy rate of 1498 hysterectomies or myomectomies with morcellation: a retrospective single-arm study. J Obstet Gynecol India 69:188-193. https://doi.org/10. 1007/s13224-018-1190-9

16. Multinu F, Casarin J, Hanson KT et al (2018) Practice patterns and complications of benign hysterectomy following the FDA statement warning against the use of power morcellation. JAMA Surg 153. https://doi. org/10.1001/jamasurg.2018.0141

\section{Publisher's Note}

Springer Nature remains neutral with regard to jurisdictional claims in published maps and institutional affiliations.

\section{Submit your manuscript to a SpringerOpen ${ }^{\circ}$ journal and benefit from:}

- Convenient online submission

- Rigorous peer review

- Open access: articles freely available online

- High visibility within the field

Retaining the copyright to your article

Submit your next manuscript at $\boldsymbol{\nabla}$ springeropen.com 